\title{
Priming of $D_{1}$-Dopamine Receptor Responses: Long-Lasting Behavioral Supersensitivity to a $D_{1}$-Dopamine Agonist Following Repeated Administration to Neonatal 6-OHDA-Lesioned Rats
}

\author{
Hugh Criswell, Robert A. Mueller, and George R. Breese \\ Biological Sciences Research Center and Mental Health Clinical Research Center, Departments of Psychiatry, \\ Anesthesiology, and Pharmacology, School of Medicine, University of North Carolina at Chapel Hill, Chapel Hill, North \\ Carolina 27599
}

The present study demonstrates that repeated administration of SKF-38393, a $D_{1}$-dopamine agonist, is necessary for maximal behavioral supersensitivity of $D_{1}$-dopamine receptor responses in neonatal 6-OHDA-lesioned rats, confirming earlier work. This repeated administration of SKF-38393, which is referred to as priming of $D_{1}$-dopamine receptor responses, resulted in a progressive increase in locomotor activity, as well as several other behaviors. This priming phenomenon lasted at least 6 months. Repeated administration of the $D_{2}$-dopamine agonist LY-171555 also increased behavioral responses to the $D_{1}$-dopamine agonist. However, previous administration of a $D_{2}$-dopamine agonist was not necessary for priming of $D_{1}$-dopamine receptor responses, because $D_{1}$-dopamine receptor priming could be produced in the presence of a $D_{2}$-dopamine receptor antagonist. Blockade of $D_{1}$-dopamine receptors with $\mathrm{SCH}-23390$ prior to injection of SKF-38393 prevented the increasing responsiveness following repeated administration of this $D_{1}$ dopamine agonist. Selective neonatal destruction of dopamine-containing neurons produced the same result as did destruction of catecholamine-containing neurons, indicating that the noradrenergic system is not involved in this phenomenon. Priming of $D_{1}$-dopamine receptor responses by repeated administration of SKF-38393 was not observed in unlesioned controls or in rats that received catecholaminedepleting lesions as adults. Repeated administration of scopolamine also was able to prime behavioral responses to SKF-38393 in neonatal 6-OHDA-lesioned rats, indicating that endogenous release of dopamine can prime $D_{1}$-dopamine receptor responses in neonatally lesioned rats. In addition, responses to indirect-acting agonists were enhanced in rats that had been primed with $\mathrm{a}_{1}$-dopamine agonist when compared with responses in unprimed animals. Furthermore, repeated microinjection of SKF-38393 into the nucleus accumbens progressively increased locomotor activity, indicating that a local action of SKF-38393 is responsible for the priming of $D_{1}$-dopamine receptor responses. Thus, priming of $D_{1}$ dopamine receptor responsiveness in neonatal 6-OHDA-le-

\footnotetext{
Received Jan. 7, 1988; revised Apr. 13, 1988; accepted May 19, 1988.

This work was supported by HD-03110 and NS-21345. We also wish to thank Carolyn Reams and Doris Lee for typing the manuscript and Edna Edwards for performing the catecholamine assays.

Correspondence should be addressed to Hugh E. Criswell, Ph.D., Biological Sciences Research Center, CB\# 7250, BSRC, University of North Carolina, Chapel Hill, NC 27599-7250.

Copyright (C) 1989 Society for Neuroscience $0270-6474 / 89 / 010125-09 \$ 02.00 / 0$
}

sioned rats represents an example of a robust, chemically specific change in neural function that is relatively permanent. The potential relationship of this phenomenon to chronic drug treatment, permanent neural messages, and symptoms in Lesch-Nyhan disease is discussed.

The $D_{1}$-dopamine agonist SKF-38393 does not produce the profound effects on behavior in normal rats that are observed after $D_{2}-$ or mixed $D_{1} / D_{2}$-dopamine agonists (Stoof and $\mathrm{Ke}$ babian, 1984; Breese et al., 1985a, b). However, following neonatal, but not adult, 6-OHDA lesions, rats exhibit greater behavioral supersensitivity to a $D_{1}$-dopamine agonist than to a $\mathrm{D}_{2}$-dopamine agonist (Breese et al., 1985a, b, 1986). Breese et al. $(1985 b)$ reported that it was necessary to administer the $D_{1}$ dopamine agonist SKF-38393 repeatedly in order to obtain maximal supersensitivity of the $D_{1}$-dopamine receptor subtype in neonatal 6-OHDA-lesioned rats. This progressive change in sensitivity with repeated treatment was referred to as "priming." Repeated administration of the $\mathrm{D}_{2}$-dopamine agonist LY-171555 (Tsuruta et al., 1981) was also capable of enhancing the effect of subsequent administration of SKF-38393, but not to the degree seen after repeated doses of SKF-38393 (Breese et al., 1985b). Recently, Morelli et al. (1987) found that the dopamine agonist-induced rotation observed in rats with unilateral 6-OHDA lesions was not seen on the first administration of a $\mathrm{D}_{1}$-dopamine agonist. While first suggesting that previous activation of the $D_{2}$-dopamine receptor was essential for demonstration of $D_{1}$-dopamine supersensitivity (Morelli et al., 1987), these workers later reported that either $D_{1}$ - or $D_{2}$-dopamine agonists could prime the $D_{1}$-dopamine receptor response after unilateral 6-OHDA lesions of the nigrostriatal pathway (Morelli and Di Chiara, 1987). The increasing sensitivity of $D_{1}$-dopamine receptors following repeated exposure to dopamine agonists suggests that the $D_{1}$-dopamine receptor may possess a degree of plasticity that could have important implications for understanding long-term changes in behavior associated with repeated drug use or the basis of symptoms of childhood disorders secondary to a central dopamine deficiency.

In the original description of priming, the neonatal 6-OHDAlesioned rats received the mixed $\mathrm{D}_{1} / \mathrm{D}_{2}$-dopamine receptor agonist, L-DOPA, prior to the administration of SKF-38393 (Breese et al., 1985b). Therefore, it was important to determine whether the priming of the $D_{1}$-dopamine receptor response in neonatal 6-OHDA-lesioned rats could be obtained without previous exposure to a dopamine agonist that influenced $\mathrm{D}_{2}$-dopamine receptors. Additionally, a variety of other aspects concerning this 
phenomenon needed to be addressed. For example, the duration of the effect had not been studied. Since both noradrenergic and dopaminergic neurons were destroyed in the rats used in early investigations, another concern was the contribution the absence of noradrenergic fibers might be having to the priming phenomenon. It was also important to know whether a classical conditioning phenomenon, whereby drug administration is associated with the testing chamber, could account for the priming effect, as has been observed for behavioral sensitization to stimulant drugs on some (Tilson and Rech, 1973), though not all (Robinson, 1984), occasions. The initial work on priming of $\mathrm{D}_{1^{-}}$ dopamine receptors measured activity (Breese et al., 1985b). It was not determined whether other behaviors associated with $\mathrm{D}_{1}$-dopamine receptors (Breese et al., 1985a) were enhanced with repeated dosing of the $\mathrm{D}_{1}$-dopamine agonist. In addition, it is important to know whether endogenous release of dopamine could prime the $D_{1}$-dopamine receptor response and if priming could be elicited by stimulation of an isolated brain region or whether more integrated CNS function was required. The function of the present investigation was to answer these questions about the increasing supersensitivity of $D_{1}$-dopamine receptors in neonatal 6-OHD 4 -lesioned rats repeatedly dosed with $\mathrm{D}_{1}$ or $\mathrm{D}_{2}$-dopamine agonists. Results from these rats were compared with those from adult 6-OHDA-lesioned and unlesioned control rats when appropriate.

\section{Materials and Methods}

\section{General}

Adult male and female Sprague-Dawley rats were obtained from Charles River Laboratories Inc. (Raleigh, NC). These animals were treated intracisternally with 6 -OHDA ( $200 \mu \mathrm{g}$ free base $/ 25 \mu \mathrm{l}) 30 \mathrm{~min}$ after 50 $\mathrm{mg} / \mathrm{kg}$ pargyline. One week later, they received an additional $200 \mu \mathrm{g}$ of 6-OHDA intracisternally (Breese and Traylor, 1970). All animals were allowed at least 2 weeks to recover from the adipsia and aphagia that can follow 6-OHDA lesioning of catecholamine-containing neurons (Breese et al., 1975). In addition to these adult rats, pregnant SpragueDawley rats obtained from Charles River Laboratories were individually housed with Wayne Lab Blox laboratory chow, and water was available ad $l i b$ until delivery. At day 3 after delivery, each neonate was anesthetized with ether and injected intracisternally with $100 \mu \mathrm{g}$ (free base) of 6-OHDA hydrobromide (Regis Chemical Co., Chicago) dissolved in $10 \mu$ of saline (Breese et al., 1975). Neonates of both sexes were then returned to their mothers with liter size limited to 10 . Some neonates were pretreated with $20 \mathrm{mg} / \mathrm{kg}$ desipramine (Regis Chemical Co.) $1 \mathrm{hr}$ prior to 6-OHDA injection to restrict the neuronal damage to dopaminecontaining neurons (Smith et al., 1973). Rats treated with 6-OHDA or saline neonatally were weaned at day 30 and testing was begun at 40 $50 \mathrm{~d}$ of age.

\section{Drugs}

The 6-OHDA hydrobromide (Sigma Chemical Co., St. Louis) was dissolved in saline containing $0.5 \%$ ascorbic acid and administered intracisternally. SKF-38393 (2,3,4,5-tetrahydro-7,8-dihydroxy-1-phenyl-1 H3-benazcpinc $\mathrm{HCl}$; Smith Kline and French Laboratories, Philadephia), theophylline (Sigma), scopolamine hydrochloride (Sigma), $d$-amphetamine sulfate (Sigma), pargyline hydrochloride (Sigma), and LY-171555 [trans-(-)-4aR-4,4a, 5, 6, 7, 8, 8a,9-octahydro-5-propyl- $1 \mathrm{H}$-Pyrazolo $(3,4)$ ] quinoline $\mathrm{HCl}$; Eli Lilly $\mathrm{Co}$., Indianapolis, IN] were dissolved in saline and administered intraperitoneally or by microinjection into the nucleus accumbens. Haloperiodol (McNeil Laboratories, Fort Washington, PA) was dissolved in $0.1 \%$ tartaric acid and administered intraperitoneally.

\section{Evaluation of behavior and activity}

Behavioral observations. Rats were placed in clear plastic cages $(23 \times$ $44 \mathrm{~cm}$ ) for $60 \mathrm{~min}$ before administration of a dopamine agonist. Once an agonist was administered, observations were made for 1 min of every $10 \mathrm{~min}$ period for $2 \mathrm{hr}$. Each $1 \mathrm{~min}$ period was divided into four $15 \mathrm{sec}$ intervals, and behaviors from a checklist were scored for occurrence in each of the $15 \mathrm{sec}$ periods. Each behavior had a maximum score of 4 for the 1 min observation period $\times 12$ observation periods, for a total of 48 (see Breese et al., 1985a). Behavior is reported as a percentage of the total possible. Behaviors monitored included sniffing, rearing, grooming, head nodding, locomotion, taffy pulling (repeated movement of the paws toward and then away from the snout), paw treading, licking, jumping, digging, eating wood chips, and self-biting (biting an extrcmity without breaking the skin). SMB was scored if biting resulted in a break in the skin, and SCH-23390 was administered at that point to block the SMB.

Activity. Activity was measured by placing rats in a circular photocell activity monitor (Hollister et al., 1974). Six photo sensors were placed about the periphery, and counts from each sensor were collected by a microcomputer and recorded at $10 \mathrm{~min}$ intervals for $150 \mathrm{~min}$. Rats were habituated to the activity chamber for $1 \mathrm{hr}$ before administration of the dopamine agonist.

\section{Microinjection procedure}

Under Nembutal anesthesia $(30 \mathrm{mg} / \mathrm{kg}$ for neonatally lesioned animals and $50 \mathrm{mg} / \mathrm{kg}$ for adult 6 -OHDA-lesioned animals and unlesioned controls), animals were prepared for microinjection by implanting a 26 gauge cannula bilaterally aimed at a site $1.5 \mathrm{~mm}$ above the nucleus accumbens $(\mathrm{AP}=2.2 \mathrm{~mm}, \mathrm{~L}=1.4 \mathrm{~mm}, \mathrm{D}=4.0 \mathrm{~mm}$; Paxinos and Watson, 1982). At least a week was allowed for recovery from the implant surgcry beforc bchavioral testing. Microinjection of SKF-38393 ( $1 \mu \mathrm{g}$ in $0.5 \mu \mathrm{l}$ of saline) was made over a $5 \mathrm{~min}$ period with the injection cannulae left in place for an additional $1 \mathrm{~min}$ following the injection. The injection cannula was 33 gauge and extended $1.5 \mathrm{~mm}$ below the tip of each guide cannula. Fluid was pumped by a Sage syringe pump through a 20-30 cm length of PE-10 tubing (see Breese et al., 1987). The location of each cannula was verified at the end of the study by examination of frozen sections.

\section{Monoamine assessment}

For catecholamine depleted animals that received drug microinjection into nucleus accumbens, the dopamine levels were determined in olfactory tubercles. Nucleus accumbens or striatal tissue was not available following histological verification of the microinjection sites. For these rats, the olfactory tubercles were dissected from the brain, weighed and placed on dry ice. Samples were stored at $-70^{\circ} \mathrm{C}$ until monoamines were determined using reverse-phase high performance liquid chromatography with electrochemical detection (Kilts et al., 1981). The remaining catecholamine depleted animals were retained and used in other studies (Duncan et al., 1987; Criswell et al., 1988). For rats with specific dopamine depleting lesions (desipramine plus 6-OHDA), samples were taken from nucleus accumbens, caudate, olfactory tubercles, and hippocampus. Dopamine and norepinephrine were determined for hippocampus by the method of Widerlov et al. (1982). Levels of dopamine, 5-HT and their major metabolites were obtained for the remaining specific sites by the method of Kilts et al. (1981). Norepinephrine was determined in brain remaining after dissection of specific regions, using the procedure of Breese and Traylor $(1970,1972)$.

\section{Statistical evaluation}

Previous work has shown that there are no significant behavioral differences between male and female 6-OHDA-lesioned rats (Breese et al., 1984). Therefore, data were collapsed across sexes for analysis. For behavioral observations, each behavior was analyzed separately using a 1-factor repeated-measures ANOVA. The scores analyzed were the percentage of $15 \mathrm{sec}$ observation periods during which a given behavior occurred (Breese et al., 1984; Criswell et al., 1988). Activity was analyzed by summing the activity counts for $150 \mathrm{~min}$ and performing a repeatedmeasures ANOVA on the totals. When the ANOVA showed a significant overall effect, individual comparisons among means were made with the Tukey HSD or Dunnett test (Winer, 1971). Progressive increases or decreases in activity over repeated testing sessions were assessed by linear trend analysis (Winer, 1971). A significant linear trend was interpreted as indicating a progressive change over time. 


\section{Results}

Measurement of activity and other behaviors following repeated administration of $S K F-38393$ to neonatal 6-OHDA-lesioned rats

The progressive increase in activity following repeated SKF38393 administration to neonatal 6-OHDA-lesioned rats is shown in Figure 1. Neither adult 6-OHDA-lesioned nor unlesioned control animals showed the progressive increase in activity levels with repeated administration of SKF-38393 observed in neonatally lesioned animals (Fig. 1). On retesting a group of 4 animals with $3 \mathrm{mg} / \mathrm{kg}$ SKF-38393 6 months after their last drug treatment, they showed a mean activity level of $18,752 \pm 5209$, which was similar to the rate observed 1 week after the last priming dose of the $D_{1}$-dopamine agonist $(19,216$ \pm 4386 counts $/ 150 \mathrm{~min} ; p>0.1$ ). Priming was not observed in a group of rats that received 5 weekly injections of saline.

The increased sensitivity to SKF-38393 following multiple administrations to neonatal 6-OHDA-lesioned rats also occurred in an observational setting (Fig. 2). There was a progressive change in locomotor activity, rearing, sniffing, digging, grooming, and paw treading following repeated administration of SKF-38393 (Fig. 2). None of these behavioral changes occurred in nonlesioned controls, adult-lesioned animals, or neonatally lesioned animals receiving repeated saline injections (see Fig. 2). An unexpected finding was the progressive decrease in selected behaviors (locomotion, sniffing, and rearing) during repeated administration of SKF-38393 to unlesioned animals (see Fig. 2). An expanded listing of behaviors after repeated administration of SKF-38393 to neonatal 6-OHDA-lesioned rats is summarized in Table 1. Greater than $90 \%$ depletion of dopamine was obtained in the olfactory tubercles of both the adult and neonatal 6-OHDA-lesioned animals (Fig. 1 legend).

Effect of environment on the progressive increase in responding after repeated administration of SKF-38393

The animals that received their initial 5 SKF-38393 injections in an environment different from the activity cages (i.e., the cages used for open-field observation) showed high levels of activity after the sixth injection when first placed in the activity test chamber (see Table $2 A$ ). This increase was comparable to that seen after multiple treatments in the activity cages (Fig. 1). Increased behavioral sensitivity to SKF-38393 was also observed when neonatally lesioned animals were treated for 5 trials in the activity chambers and subsequently tested in the observation chambers on trial 6 (Table $2 B$ ). It is also important to note that the activity response to the first dose of SKF-38393 after animals had been tested for 5 trials with saline injections did not differ from that of rats naive to the activity chamber (Fig. 1 legend).

Investigation of the priming of $D_{1}$-dopamine receptor responses in rats with preferential reduction of dopamine

Repeated administration of the $\mathrm{D}_{1}$-dopamine agonist SKF-38393 to rats treated neonatally to destroy selectively only dopaminccontaining neurons (Smith et al., 1973) also was found to produce a progressive increase in activity (Fig. 3), similar to that seen in rats that had both dopamine- and norepinephrine-containing fibers destroyed (Fig. 1). Behavioral responses were also increased in this treatment group after repeated administration of SKF-38393 (Table 3). Documentation of the effectiveness of
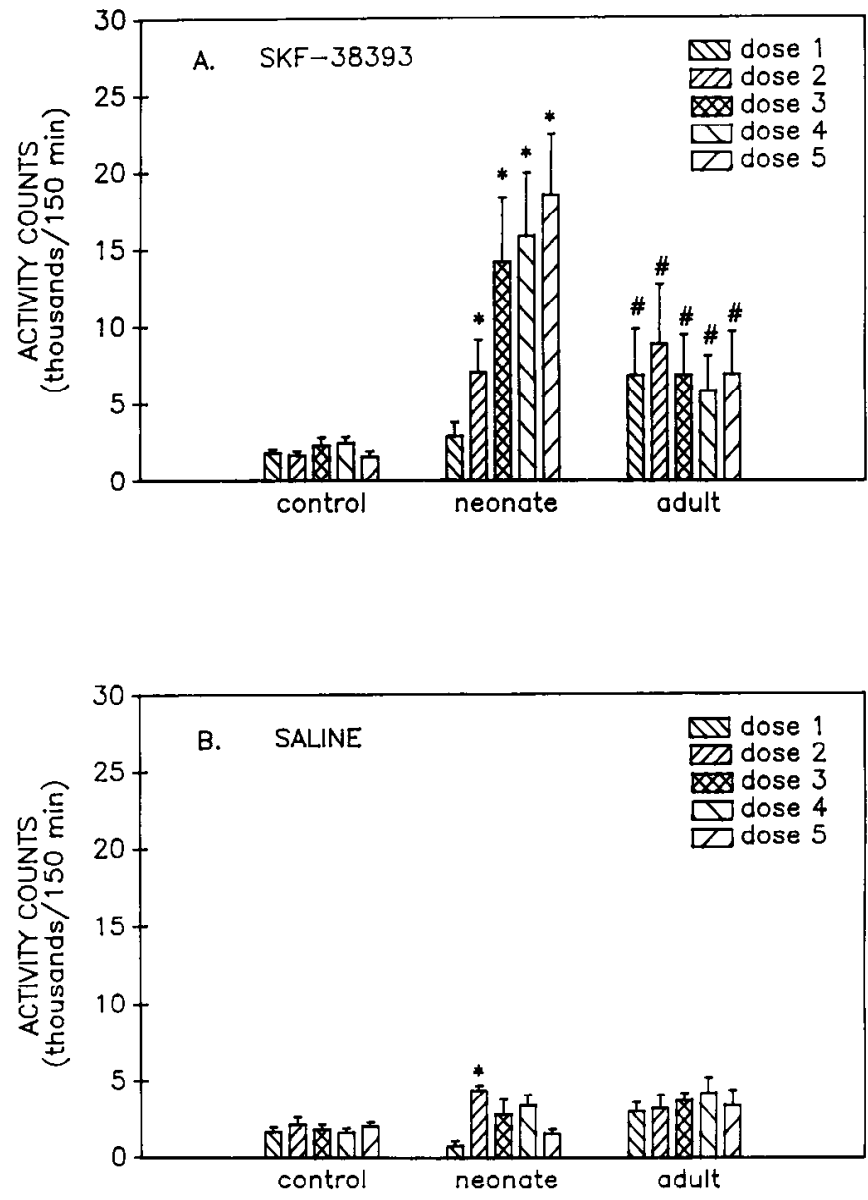

Figure 1. Locomotor activity following repeated weekly administration of the $D_{1}$-dopamine agonist SKF-38393 $(A)$ or saline $(B)$ to unlesioned, neonatal and adult 6-OHDA-lesioned rats. All animals were tested as adults. Dose refers to treatments with either saline or SKF38393 (3 mg/kg, i.p.) at 1 week intervals. When SKF-38393 was administered for the first time to the neonatal 6-OHDA-lesioned rats following the 5 saline injections in $B$, the activity response was $4545 \pm$ 756 counts $/ 150 \mathrm{~min} \pm \operatorname{SEM}(p>0.1$ compared with dose 1 to neonatal 6-OHDA-lesioned rats in $A$ ). The linear trend for increases in locomotion with repeated administration of SKF-38393 to neonate 6-OHDAlesioned rats was significant $(p<0.01)$. There were no other significant linear trends $(p>0.1)$. Dopamine content of olfactory tubercles was $6.0+0.3 \mathrm{ng} / \mathrm{mg}$ protein in control animals, $0.4 \pm 0.1 \mathrm{ng} / \mathrm{mg}$ protein in neonatal 6-OHDA-lesioned rats, and $0.3 \pm 0.05 \mathrm{ng} / \mathrm{mg}$ protein in adult 6-OHDA-lesioned rats. Depletion in the olfactory tubercles was $93 \%$ in neonatally treated animals and $96.5 \%$ in adult treated animals compared with controls. ${ }^{*} p<0.05$ compared with day $1 ; \# p<0.05$ compared with unlesioned controls.

the 6-OHDA treatment to reduce only dopamine is provided in the legend of Figure 3.

Investigation of the possible involvement of $D_{2}$-dopamine receptors in the priming of $D_{1}$-dopamine receptor responses

When the $\mathrm{D}_{2}$-dopamine antagonist haloperidol was coadministercd with the $D_{1}$-dopamine agonist, the SKF-38393-induced activity response did not differ from the response observed in the absence of haloperidol (Fig. 4). On the other hand, prior administration of the $\mathrm{D}_{1}$-dopamine antagonist, $\mathrm{SCH}-23390$ (Iorio et al., 1983), antagonized the response to SKF-38393 and prevented the priming of the $D_{1}$-dopamine receptor response (Fig. 4). When prior administration of SCH-23390 was halted, 
Figure 2. Behavioral changes following repeated weekly administration of the $D_{1}$-dopamine agonist SKF-38393. NEO-SKF, AD-SKF, and CON-SKF refer to SKF-38393 administration to neonatal 6-OHDA-lesioned, to adult 6-OHDA-lesioned, and to unlesioned rats, respectively; NEO-SAL, AD-SAL, CON-SAL refer to saline administration to these latter groups. Dose refers to the weekly administration of SKF38393 ( $3 \mathrm{mg} / \mathrm{kg}$ ) for 5 weeks. See Materials and Methods for definition of percent of time. NEO-SKF animals showed significant linear trends for increasing levels of locomotor activity, rearing and sniffing, while CON-SKF animals showed significant linear trends for decreases in these behaviors with successive doses. ${ }^{*} p<0.05$ compared with day 1 .
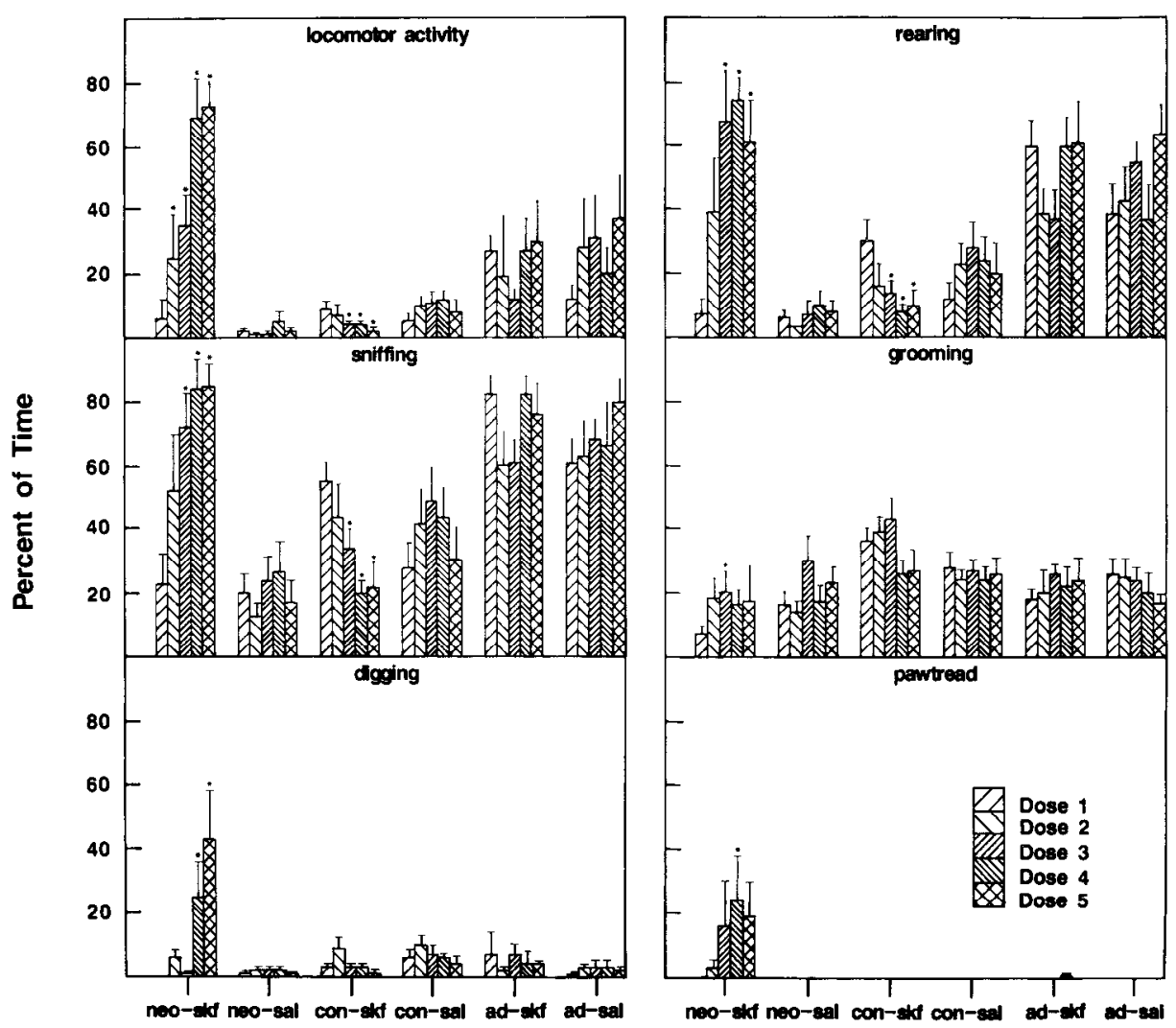

subsequent administration of SKF-38393 caused a progressive increase in responding, demonstrating the ability of these rats to manifest priming of the $D_{1}$-dopamine receptor response (Fig. 4).

Repeated administration of the selective $\mathrm{D}_{2}$-dopamine agonist LY-171555 did not result in a significant increase in the sensitivity to the $\mathrm{D}_{2}$-dopamine agonist but did result in an increase in the subsequent response to a $D_{1}$-dopamine agonist (Table 4). The magnitude of the increase in responsiveness to SKF-38393 after LY-171555 was less than that seen after SKF38393. However, as apparent from the SEM of this group, there was considerable variability in the degree to which the LY171555 would alter the response to a subsequent dose of SKF38393.

Examination of priming of $D_{1}$-dopamine receptors following microinjection of SKF-38393 into the nucleus accumbens

A progressive increase in activity was observed following repeated microinjection of $1 \mu \mathrm{g}$ of SKF-38393 into the nucleus accumbens of neonatal 6-OHDA-lesioned, but not of nonlesioned control, animals. This increased activity level observed after repeated microinjection into the nucleus accumbens was

Table 1. Effect of repeated doses of SKF-38393 on behavior in control and neonatal 6-OHDA-lesioned rats

\begin{tabular}{|c|c|c|c|c|c|}
\hline \multirow[b]{3}{*}{ Behavior } & \multirow{3}{*}{$\begin{array}{l}\text { Saline } \\
\text { 6-OHDA } \\
(n=9)\end{array}$} & \multicolumn{4}{|c|}{ Percentage incidence with SKF-38393 } \\
\hline & & \multicolumn{2}{|l|}{ Dose 1} & \multicolumn{2}{|c|}{ Dose 5 or $6^{a}$} \\
\hline & & Control & 6-OHDA & Control & 6-OHDA \\
\hline Quiet & $69 \pm 6$ & $22 \pm 4^{c}$ & $54 \pm 10$ & $54 \pm 9$ & $7 \pm 4^{b . c}$ \\
\hline Locomotion & $2 \pm 1$ & $9 \pm 2^{c}$ & $5 \pm 2$ & $1 \pm 1^{h}$ & $52 \pm 10^{b, c}$ \\
\hline Sniffing & $20 \pm 6$ & $55 \pm 6^{c}$ & $40 \pm 9$ & $24 \pm 8^{b}$ & $78 \pm 6^{b, c}$ \\
\hline Rearing & $6 \pm 2$ & $30 \pm 5^{c}$ & $10 \pm 4$ & $11 \pm 5^{h}$ & $48 \pm 10^{b, c}$ \\
\hline Paw treading & $0 \pm 0$ & $0 \pm 0$ & $13 \pm 8$ & $0 \pm 0$ & $12 \pm 7$ \\
\hline Grooming & $16 \pm 3$ & $36 \pm 4^{c}$ & $5 \pm 1^{c}$ & $29 \pm 6^{c}$ & $19 \pm 7^{b}$ \\
\hline Digging & $0 \pm 0$ & $4 \pm 1$ & $3 \pm 3$ & $1 \pm 1$ & $36 \pm 12^{b, c}$ \\
\hline Cage licking & $0 \pm 0$ & $0 \pm 0$ & $0.4 \pm 0.3$ & $0 \pm 0$ & $2 \pm 2$ \\
\hline Taffy pulling & $0 \pm 0$ & $0 \pm 0$ & $3 \pm 2$ & $0 \pm 0$ & $0.2 \pm 0.0$ \\
\hline Self-biting & $0 \pm 0$ & $0 \pm 0$ & $4 \pm 2$ & $0 \pm 0$ & $0 \pm 0$ \\
\hline
\end{tabular}

a There are 13 rats in Dose 1 group data and 10 rats in the Dose 5 or 6 group data. SKF-38393 (3 mg $/ \mathrm{kg})$ was administered 5 or 6 times at weekly intervals and behavioral responses noted as described in Materials and Methods.

${ }^{b} p<0.05$ compared with Dose 1 response for that drug.

' $p<0.05$ compared with saline. 


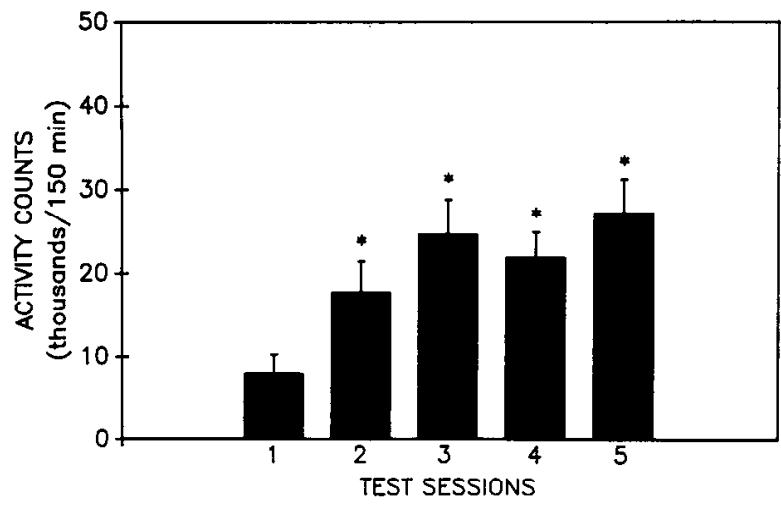

Figure 3. Effect of repeated administration of SKF-38393 on activity level in rats with selective neonatal destruction of dopamine-containing neurons. The linear trend for increased responding with repeated administration was significant $(p<0.01)$. Monoamine concentrations in 6-OHDA-lesioned rats treated to reduce only dopamine were as follows: Dopamine was reduced in olfactory tubercles from $24.90 \pm 1.30 \mathrm{ng} /$ $\mathrm{mg}$ protein to $2.8 \pm 0.6 \mathrm{ng} / \mathrm{mg}$ protein $(89 \%)$. Dopamine was reduced from $65.6 \pm 3.9 \mathrm{ng} / \mathrm{mg}$ and $56.5 \pm 8.4 \mathrm{ng} / \mathrm{kg}$ protein, in the nucleus accumbens or caudate respectively in controls to no detectable content of dopamine ( $>99 \%$ depletion) in lesioned animals. Norepinephrine levels were not significantly changed (3\% increase in hippocampus from $11.6 \pm 1.9$ to $12.0 \pm 2.0 \mathrm{ng} / \mathrm{mg}$ protein and $10 \%$ depletion in the rest of the brain from $65.6 \pm 3.0$ to $58.0 \pm 3.0 \mathrm{ng} / \mathrm{mg}$ protein; $p>0.1$ ). 5 -HT was increased in 6-OHDA-lesioned rats from $3.6 \pm 0.2$ (control) to $12.6 \pm 0.4 \mathrm{ng} / \mathrm{mg}$ protein in the caudate nucleus $(p<0.05)$ but was unchanged in nucleus accumbens and olfactory tubercles $(4.2 \pm 0.5$ vs $4.8 \pm 0.5$ and $7.2 \pm 0.3$ vs $8.2 \pm 0.8 \mathrm{ng} / \mathrm{mg}$ protein, respectively; $p>$ $0.05)$ in control and lesioned rats. ${ }^{*} p<0.05$ compared with the first dose.

Table 2. Effect of environment on subsequent responses to $D_{1^{-}}$ dopamine agonist administration

A. Locomotor activity after open-field testing ${ }^{\prime \prime}$

Locomotor activity

SKF-38393 dose

(counts/150 min $\pm \mathrm{SEM}$ )

$1 \quad 2442 \pm 760$

$619,698 \pm 5890^{h, c}$

B. Behavioral testing in open field after priming in activity monitors SKF-38393

\begin{tabular}{lrl} 
Behavior & \multicolumn{1}{c}{ Dose 1 } & Dose 6 \\
\hline Quiet & $75.0 \pm 8.0$ & $25.1 \pm 12.0^{\circ}$ \\
Locomotion & $5.6 \pm 4.8$ & $26.2 \pm 7.2^{e}$ \\
Sniffing & $22.9 \pm 8.9$ & $71.7 \pm 6.7^{e}$ \\
Rearing & $7.3 \pm 5.2$ & $38.3 \pm 13.9^{\circ}$ \\
Paw treading & $0.0 \pm 0.0$ & $15.4 \pm 14.9$ \\
Grooming & $7.0 \pm 2.0$ & $20.0 \pm 5.0^{e}$ \\
Digging & $0.0 \pm 0.0$ & $19.0 \pm 15.0$
\end{tabular}

a Activity counts were recorded in a circular activity chamber. The Dose 1 group had not previously received SKF-38393, while the Dose 6 group had received 5 previous injections of SKF-38393 in a different environmental setting (behavioral observation cages).

${ }^{b} p<0.01$ compared with dose 1 .

' $p>0.1$ compared with dose 6 in rats dosed only in activity cages.

${ }_{d}$ Neonatal 6-OHDA-lesioned animals were injected with $3 \mathrm{mg} / \mathrm{kg}$ of SKF-38393, placed in clear plastic cages and observed for $2 \mathrm{hr}$. The Day 1 group had not previously received SKF-38393, while the Day 6 group had received 5 injections of SKF-38393 in a different environmental setting (circular activity chamber) Numbers represent the precent of time that the animals engaged in the listed behaviors. The values on day 6 agrec favorably with those in Table 1 . There wcrc 6 rats in each group.

${ }^{\circ} p<0.05$ compared with dose 1 in the same environment.

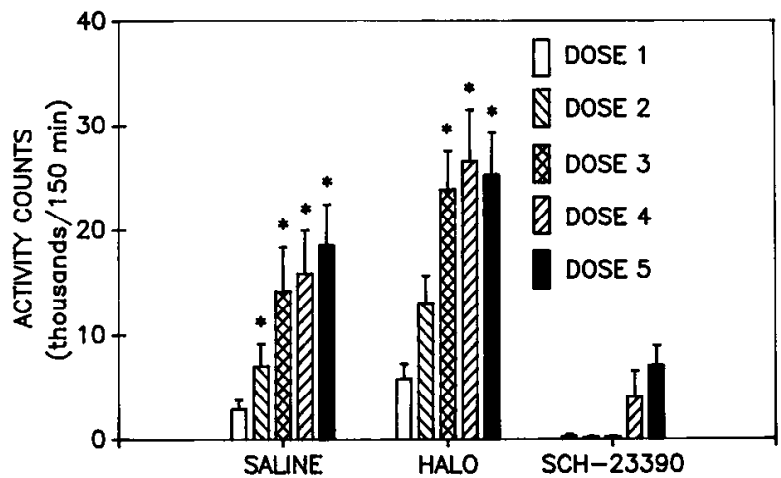

Figure 4. Priming in neonatal 6-OHDA-lesioned rats by SKF-38393 in the presence of haloperidol or SCH-23390. Neonatal 6-OHDA-lesioned rats were treated with the $\mathrm{D}_{2}$-dopamine antagonist haloperidol (HALO;0.5 mg/kg) or the $\mathrm{D}_{1}$-dopamine antagonist SCH-23390 $(0.3$ $\mathrm{mg} / \mathrm{kg}$ ) or saline $15 \mathrm{~min}$ prior to receiving $3 \mathrm{mg} / \mathrm{kg}$ of the $D_{1}$-dopamine agonist SKF-38393. A minimum of $4 \mathrm{~d}$ was allowed between successive treatments. No SCH-23390 was given prior to the SKF-38393 on the last 2 testing sessions. The linear trend for increased locomotor activity was significant in both the saline and haloperidol-pretreated group ( $p$ $<0.01)$ but not in the SCH-23390 pretreated group $(p>0.1){ }^{*} p<$ 0.05 compared with day 1 .

roughly equivalent to the response seen after systemically administered SKF-38393 (Fig. 5). While there was an apparent further increase in responsiveness observed with the repeated systemic injection of SKF-38393 in the rats repeatedly treated centrally with SKF-38393 (Fig. 5), this change did not reach statistical significance when day 1 response was compared with that produced on day $3(p<0.1)$.

\section{Evaluation of the ability of indirect-acting dopamine agonists to prime the $D$,-dopamine receptor response}

Previous data indicate that scopolamine and theophylline increase the activity level in 6-OIIDA-lesioned rats; this response is dependent upon endogenous catecholamine release (Ondrusek

\section{Table 3. Behavioral responses at dose 1 and dose 5 following repeated weekly administration of the $D_{1}$-dopamine agonist SKF- 38393 to rats with neonatal lesions to dopamine-containing neurons}

\begin{tabular}{llcc} 
& & \multicolumn{2}{c}{$\begin{array}{l}\text { Percent incidence with } \\
\text { SKF-38393 }\end{array}$} \\
\cline { 3 - 4 } Behavior & $\begin{array}{l}\text { Saline } \\
(n=9)\end{array}$ & Dose 1 & Dose 5 \\
\hline Quiet & $26 \pm 12$ & $9 \pm 4$ & $0 \pm 0^{b}$ \\
Locomotion & $32 \pm 8$ & $38 \pm 11$ & $59 \pm 10^{b}$ \\
Sniffing & $72 \pm 12$ & $75 \pm 11$ & $86 \pm 4$ \\
Rearing & $43 \pm 13$ & $46 \pm 19$ & $60 \pm 10$ \\
Paw treading & $6 \pm 3$ & $0 \pm 0$ & $35 \pm 10^{a . b}$ \\
Grooming & $5 \pm 1$ & $24 \pm 7^{b}$ & $24 \pm 8^{b}$ \\
Digging & $0 \pm 0$ & $1 \pm 1$ & $30 \pm 8^{a . b}$ \\
Cage licking & $0 \pm 0$ & $0 \pm 0$ & $0 \pm 0$ \\
Taffy pulling & $0 \pm 0$ & $0 \pm 0$ & $13 \pm 8$ \\
Self-biting & $0 \pm 0$ & $0 \pm 0$ & $4 \pm 4$
\end{tabular}

Rats were treated as neonates to reduce only brain dopamine (see Materials and Methods). When adult, rats were given 5 doses of SKF-38393 $(3 \mathrm{mg} / \mathrm{kg})$ at weekly intervals and behavior was observed in the open-field containers. Representative results from days 1 and 5 are presented. The saline group includes 9 rats, dose 1 includes 6 rats and dose 5 includes 10 rats. Values for control rats after 1 and 5 doses of SKF-38393 are given in Table 1 .

${ }^{a} p<0.05$ compared with dose 1 .

${ }^{n} p<0.05$ compared with saline. 


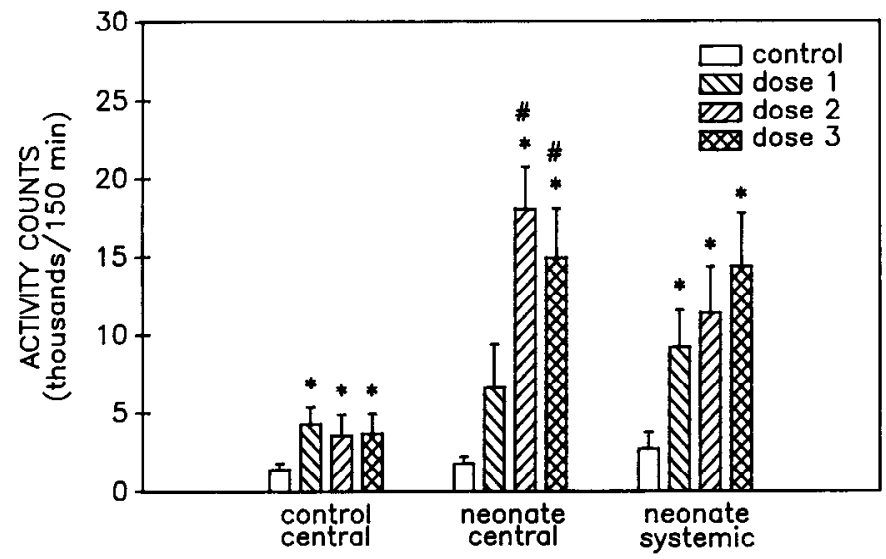

Figure 5. Activity response after repeated microinjection of $1 \mu \mathrm{g}$ of SKF-38393 into the nucleus accumbens (central) of neonatal 6-OHDAlesioned rats (neonate) or unlesioned control (control) animals followed by systemic administration (systemic) of SKF-38393 to neonatally lesioned rats or to unlesioned control animals. Following the 3 microinjections of SKF-38393 into the nucleus accumbens (central), subsequent systemic doses (systemic) of SKF-38393 (3 mg/kg, i.p.) were administered to the groups that received microinjections into the nucleus accumbens. Treatments (Dose) were at 1 week intervals. There are at least 5 rats in each group. ${ }^{*} p<0.05$ compared with saline; $\# p<0.05$ compared with naive animals.

et al., 1981; Criswell et al., 1988). Therefore, we chose to use these indirect-acting dopamine agonists to see if endogenous release of dopamine would prime $D_{1}$-dopamine receptor responses. In initial experiments, scopolamine and theophylline were repeatedly administered to neonatal 6-OHDA-lesioned rats at weekly intervals for 3 doses (Fig. 6). Following this treatment, the response to SKF-38393 was enhanced in the rats that received scopolamine (Fig. 6). There was a strong tendency for the response to be elevated after theophylline, but this did not reach statistical significance (Fig. 6).

\section{Effect of previous priming of $D_{I}$-dopamine receptor responses on the responses to indirect-acting dopamine agonists}

Previous priming of the $\mathrm{D}_{1}$-dopamine receptor response with SKF-38393 resulted in an increased activity level following administration of theophylline or scopolamine (Fig. 7). Furthermore, the decreased ability of $d$-amphetamine to stimulate locomotor activity after 6-OHDA lesions was partially reversed by priming of the $\mathrm{D}_{1}$-dopamine receptor response (Fig. 7).

\section{Table 4. Effect of repeated administration of LY-171555 on subsequent response to SKF-38393}

\begin{tabular}{llc}
$\begin{array}{l}\text { Dopamine agonist } \\
\text { dose }^{a}\end{array}$ & $\begin{array}{l}\text { No. of } \\
\text { rats tested }\end{array}$ & $\begin{array}{l}\text { Activity response } \\
\text { (counts/150 min } \pm \\
\text { SEM) }\end{array}$ \\
\hline LY-171555-1 & 10 & $7282 \pm 1583$ \\
LY-171555-2 & 10 & $9353 \pm 1665$ \\
LY-171555-3 & 10 & $10,473 \pm 1916$ \\
SKF-38393-1 & 10 & $13,710 \pm 4483^{b}$ \\
SKF-38393-2 & 6 & $15,732 \pm 6076^{b}$
\end{tabular}

a Animals received $\mathrm{LY}-171555(\mathrm{l} \mathrm{mg} / \mathrm{kg})$ doses at weekly intervals. After 3 doses of LY-171555, rats were given SKF-38393 (3 mg/kg). The responses to different doses of LY-171555 do not differ $(p>0.1)$. The first dose of SKF-38393 produced an activity response of $2894 \pm 980$ counts $/ 150 \mathrm{~min}$ in a saline-treated group.

${ }^{b} p<0.01$ compared with dose 1 of SKF-38393 in naive 6-OHDA-lesioned neonates.

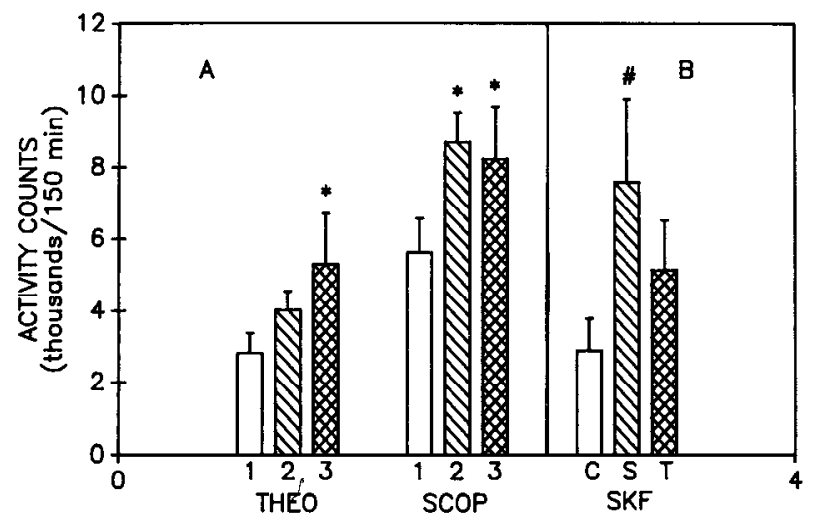

Figure 6. Effect of repeated administration of theophyllinc and scopolamine on the subsequent activity response to SKF-38393. $A$, The doses of theophylline (THEO; $10 \mathrm{mg} / \mathrm{kg}$, i.p.) and scopolamine (SCOP; $0.5 \mathrm{mg} / \mathrm{kg}$, i.p.) were administered at weekly intervals for 3 weeks and activity was measured. $B$, One week after the third dose of scopolamine $(S)$ or theophylline $(T)$ SKF-38393 $(3 \mathrm{mg} / \mathrm{kg})$ was administered and responses to $\mathrm{SKF}-38393$ were compared with responses from naive control $(C)$ animals. ${ }^{*} p<0.05$ compared with response in saline; \# $p$ $<0.05$ compared with response in naive rats.

\section{Discussion}

As previously reported (Breese et al., 1985b), repeated weekly administration of the $D_{1}$-dopamine agonist SKF-38393 to neonatal 6-OHDA-lesioned rats resulted in a rapidly escalating activity level that peaked after 3-5 treatments. Because $\mathrm{SCH}-23390$ prevented priming of the $\mathrm{D}_{1}$-dopamine receptor response, this priming phenomenon cannot be associated with a nonspecific action of SKF-38393. Priming of the $D_{1}$-dopamine receptor response appears to be permanent with supersensitive behavioral responses still present 6 months after the last priming session. At present, we do not know whether the time between treatments is an important determinant of priming as it is in behavioral sensitization to amphetamine (Robinson and Becker, 1986). Previous work performed with L-DOPA showed that a

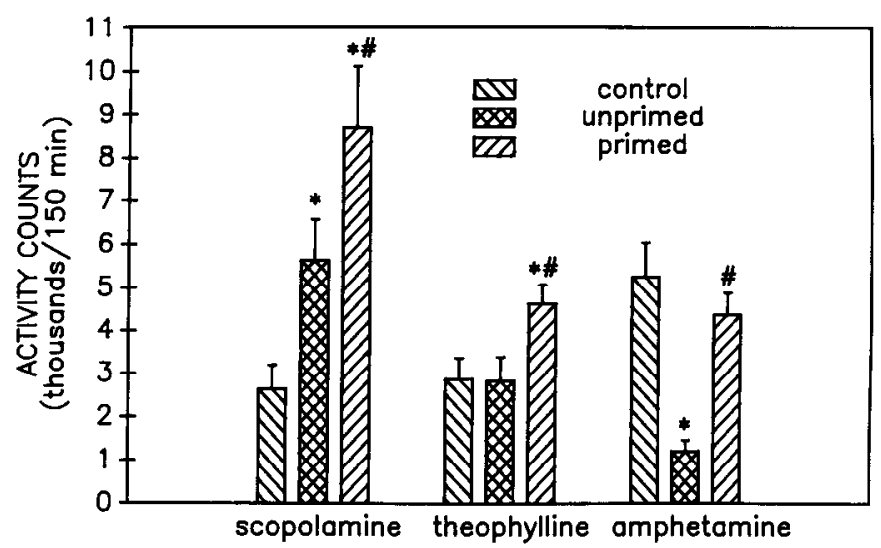

Figure 7. Administration of scopolamine, theophylline, and $d$-amphetamine to rats with and without previous priming of $D_{1}$-dopamine receptor responses. Control refers to rats, not lesions; unprimed refers to naive neonatal 6-OHDA-lesioned rats; primed refers to rats that received 5 previous treatments of SKF-38393 $(3 \mathrm{mg} / \mathrm{kg})$ at weekly intervals prior to receiving the indirect dopamine agonists. The i.p. dose for scopolamine was $0.5 \mathrm{mg} / \mathrm{kg}$, for theophylline was $10 \mathrm{mg} / \mathrm{kg}$, and for $d$-amphetamine was $1 \mathrm{mg} / \mathrm{kg}$. ${ }^{*} p<0.05$ compared with response in unlesioned controls; \# $p<0.05$ compared with response in unprimed animals. 
single large dose of L-DOPA did not produce a significant amount of priming (Breese et al., 1985a). This suggests that a larger dose of SKF-38393 would not greatly facilitate this phenomenon. These variables will be the focus of future investigations.

Priming of the $D_{1}$-dopamine receptor response was also reflected by an increase in the frequency of several other behaviors elicited by SKF-38393, including rearing, sniffing, grooming, digging, and paw treading. The behavioral responses to SKF38393 in neonatal 6-OHDA-lesioned rats have been summarized previously (Breese et al., 1985a). In general, there is a reasonable congruence between the incidence with which a behavior was observed in the present study and that noted in our previous report, except for 2 behaviors - taffy pulling and selfbiting. In the present study, the incidence of these latter behaviors after SKF-38393 was considerably lower than previously reported after SKF-38393 (Breese et al., 1985a). A difference between these studies is that the rats in the present investigation were not treated with L-DOPA prior to receiving the multiple SKF-38393 treatment (Breese et al., 1985b). Because these behaviors are frequently observed after L-DOPA administration to neonatal 6-OHDA-lesioned rats (Breese et al., 1985a), it is possible that taffy pulling and self-biting require activation of both $\mathrm{D}_{1}$ - and $\mathrm{D}_{2}$-dopamine receptors for a maximal behavioral expression. We have previously reported the importance of $D_{1} /$ $\mathrm{D}_{2}$-dopamine receptor activation to produce a maximal incidence of self-mutilatory behavior in the neonatal 6-OHDAlesioned rat, even though $\mathrm{D}_{1}$-dopamine receptors are critical for this behavior to occur (Breese et al., 1985a).

Previous work has shown that both neonatal and adult 6-OHDA-lesioned animals are supersensitive to the behavioral effects of the $D_{2}$-dopamine agonist LY-171555, with adult-lesioned animals exhibiting significantly more behavioral activation than the neonatally lesioned animals after this drug (Breese et al., 1985a, b). In agreement with earlier work (Breese et al., $1985 \mathrm{~b}$ ), the supersensitivity to $a D_{2}$-dopamine agonist was found to be present from the first treatment and did not increase with repeated dosing in the neonatal 6-OHDA-lesioned rat. While priming of the $\mathrm{D}_{2}$-dopamine receptor response was not observed with repeated administration of the $D_{2}$-dopamine agonist, this treatment resulted in priming of responses to the $D_{1}$-dopamine agonist in neonatal 6-OHDA-lesioned rats. In our earlier studies, the animals received one dose of the mixed $D_{1} / D_{2}$-dopamine agonist, L-DOPA, prior to investigating the priming response to $D_{1}$-dopamine receptor activation (Breese et al., 1985b). Morelli et al. (1987) recently reported that prior stimulation of the $\mathrm{D}_{2}$-dopamine receptor was required for subsequent supersensitivity to $D_{1}$-dopaminc agonists in adult unilatcrally lesioned rats; however, Morelli and Di Chiara (1987) subsequently reported that both $D_{1}$ - and $D_{2}$-dopamine agonists can prime $D_{1}$ dopamine receptors in animals with unilateral 6-OHDA lesions of the nigrostriatal pathway. In the present study, priming of the $\mathrm{D}_{1}$-dopamine receptor response was observed in drug-naive neonatal 6-OHDA-lesioned animals irrespective of whether or not the $\mathrm{D}_{2}$-dopamine antagonist haloperidol was administered concurrently with the $D_{1}$-dopamine agonist. Thus, even though $\mathrm{D}_{2}$-dopamine receptor activation can contribute to priming of $\mathrm{D}_{\mathrm{t}}$-dopamine receptors, the present results clearly indicate that $\mathrm{D}_{2}$-dopamine receptor stimulation is not necessary for induction or expression of behavioral supersensitivity to $D_{1}$-dopamine agonists in neonatal 6-OHDA-lesioned animals. Priming of the $\mathrm{D}_{1}$-dopamine receptor response was also observed in animals with selective destruction of dopamine-containing neurons. This indicates that absence of other catecholamine-containing neurons is not necessary for priming to occur. The behavioral profile of these selectively lesioned animals differed slightly from that of animals with nonselective catecholamine depletion, which is consistent with previous literature (Breese et al., 1984).

The critical importance of the age at which the 6-OHDA lesion is made to the subsequent response seen to dopamine agonists has becn described in previous work (Breese and Traylor, 1972; Breese et al., 1984). A relatively selective supersensitivity to $\mathrm{D}_{1}$-dopamine agonists is observed in neonatal 6-OHDA-lesioned rats, whereas adult 6-OHDA-lesioned rats exhibit a greater sensitivity to $\mathrm{D}_{2}$-dopamine agonists (Breese et al., 1985b). Neonatal 6-OHDA-lesioned rats do not exhibit the period of adipsia and aphagia associated with adult 6-OHDA lesions, even though they have lower body weights following the lesion (Breese and Traylor, 1972). Additionally, neonatally lesioned rats are subsensitive to the immobility produced by both $\mathrm{D}_{1}$ - and $\mathrm{D}_{2}$-dopamine antagonists when compared with rats lesioned as adults (Bruno et al., 1985; Duncan et al., 1987), and have an elevated content of 5-HT, a change not seen in adult-lesioned rats (Breese et al., 1984). In the present study, animals that received 6-OHDA lesions as adults did not show a progressive increase in responding to repeated administration of SKF-38393 as did the neonatally lesioned rats. Such results provide further evidence that adaptive variables in the neonatally lesioned rat differ from those seen in rats lesioned as adults.

Scopolamine and theophylline can produce enhanced activity levels in 6-OHDA-lesioned rats, and this response appears to depend on catecholamine release from remaining neurons (Ondrusek et al., 1981; Criswell et al., 1988). In neonatal 6-OHDAlesioned rats, the response to the first dose of these compounds was less than that seen after the compounds had been administered repeatedly for 3 doses. Subsequently, the response to SKF-38393 was found to be greater than that seen in naive neonatal 6-OHDA-lesioned animals receiving their first dose of this $D_{1}$-dopamine agonist. In addition, present data demonstrated that the responses to scopolamine and theophylline were greater in rats previously treated repeatedly with SKF-38393 than in drug-naive neonatal 6-OHDA-lesioned rats. Even though amphetamine is dependent upon the release of dopamine from remaining terminals and is depressed following 6-OHDA lesions, primed animals showed greater activity to amphetamine than unprimed animals. These data raise several questions. Because $d$-amphetamine is an indirect-acting dopamine agonist, it is not known why the action of $d$-amphetamine is decreased in 6-OHDA-lesioned rats, whereas the action of scopolamine and thcophyllinc is cnhanced. This would suggest differing mechanisms by which the dopamine is released. Nonetheless, it is clear that endogenous release of dopamine can prime $\mathrm{D}_{1}$ dopamine receptor responses in neonatally lesioned rats and that previous repeated exposure to a $D_{1}$-dopamine agonist can enhance the response of indirect-acting psychostimulants. Yet to be answered is whether endogenous release in unlesioned rats, if sufficiently intense, can have a similar effect on $D_{1}$ dopamine receptor responsiveness (i.e., prime the response). In the present study, at the doses of SKF-38393 repeatedly administered systemically, or directly into brain, we were unable to demonstrate priming of $\mathrm{D}_{1}$-dopamine receptor responsiveness in unlesioned rats. In fact, repeated administration of SKF38393 produced a reduction in some behaviors in unlesioned rats, suggesting that tolerance was developing. Neither priming nor tolerance was observed in adult 6-OHDA-lesioned rats. We 
presume that the proposed coupling of $D_{1}$ - and $D_{2}$-dopamine receptors through intact catecholamine-containing neurons must account for these latter observations (Breese and Mueller, 1985; Breese et al., 1985b).

Repeated administration of the indirect-acting dopamine agonists, $d$-amphetamine or cocaine, can result in behavioral supersensitivity in unlesioned rats providing that sufficient time is allowed between administrations (Post, 1980; Robinson and Becker, 1986). Therefore, it would not be surprising to find a link between the elevated responses to these psychostimulants and priming of $\mathrm{D}_{1}$-dopamine receptor responses. However, this link has yet to be made, and the available data can be used to argue for and against such a relationship. There is one component of the priming of $D_{1}$-dopamine receptor responses observed in our investigations that seems to differ from that observed with repeated administration of indirect-acting dopamine agonists. Some researchers have found that classical conditioning to the testing environment is important to the enhanced sensitivity of $d$-amphetamine (Tilson and Rech, 1973), while others have shown behavioral supersensitivity to amphetamine in the absence of conditioning cues (Robinson, 1984; Coward, 1986). The present study demonstrated that $D_{1}$-dopamine-receptor priming can be exhibited even when animals are repeatedly dosed in an environment different from that in which the rats are finally tested (Table 2). This latter observation minimizes the potential importance of conditioning to the priming of $D_{1}$-dopamine receptor responses and could imply differences with the enhanced behavioral sensitivity observed after cocaine and $d$-amphetamine. It is apparent that the increasing sensitivity of $D_{1}$-dopamine receptors with repeated administration is due to a change in postsynaptic responsivencss. If there is a changc in $\mathrm{D}_{1}$-dopamine responsiveness with chronic administration of indirect-acting compounds, it would involve a presynaptic change in dopamine release, a postsynaptic supersensitivity, or both. Robinson and Becker (1986) reported in a recent review that presynaptic mechanisms are altered after chronic $d$-amphetamine, but only 8 of 20 studies were able to implicate a change in postsynaptic receptor sensitivity. A possible reason for the apparent absence of a change in postsynaptic responsiveness in these earlier studies may relate to the general use of apomorphine to test for postsynaptic receptor sensitivity (Klawans and Margolins, 1975). Apomorphine at the doses tested in these investigations activates primarily $\mathrm{D}_{2}$-dopamine receptors (Breese et al., 1985b). In subsequent experiments, it would seem reasonable to determine $D_{1}$-dopamine receptor sensitivity after chronic $d$-amphetamine treatment using a specific $D_{1}$-dopamine agonist. However, it may be difficult to see a change in sensitivity after systemic administration of SKF-38393 in unlesioned rats. Future studies will have to resolve such difficulties before it can be determined whether the enhanced behavioral responses after repeated psychostimulant treatment are due to a change in $D_{1}$ dopamine receptor sensitivity.

Priming of the $D_{1}$-dopamine receptor responsiveness in the neonatal 6-OHDA-lesioned rat represents a model of a longterm change in neural function associated with repeated activation of a chemically defined receptor system. As such, the underlying neurochemical mechanisms that lead to this increased sensitivity may be representative of changes in other central processes requiring a "permanent" neural message. Related areas might include learning, development of craving following repeated intake of some drugs, or the appearance of motor disturbances following prolonged administration of drugs that influence dopamine receptor function.

The neonatal 6-OHDA-lesioned rat is proposed to model the dopamine depletion seen in Lesch-Nyhan disease (Breese et al., 1984). Thus, the endogenous release of dopamine from remaining neurons in this syndrome could presumably sensitize $D_{1}$-dopamine receptors, as was seen with the indirect-acting dopamine agonists in this investigation. However, there is no direct cvidence that this is the case. It is our opinion that a stress component might be necessary for this to occur (i.e., stressinduced dopamine release). We have proposed that reduced adenosine seen in Lesch-Nyhan disease could facilitate central mechanisms resulting in dopamine release (Criswell et al., 1988). Nevertheless, based upon these present animal studies, priming of $D_{1}$-dopamine receptor function could be an important component of the self-biting behavior observed in patients with Lesch-Nyhan disease (see also Breese et al., 1984, 1985b). The behavioral supersensitivity associated with the priming of $\mathrm{D}_{1}$-dopamine receptors is not associated with an increase in dopamine receptor number or affinity when evaluated with antagonist ligands (Breese et al., 1987; Duncan et al., 1987). Thus, the increased effectiveness of $D_{1}$-dopamine receptor agonists after repeated administration must, presumably, be associated either with a change in second or third messengers linked to this receptor or with a change in a system or systems modulating dopamine-mediated effects. Future research will attempt to resolve the neurochemical basis for this priming phenomenon.

\section{References}

Breese, G. R., and R. A. Mueller (1985) SCH-23390 antagonism of a $\mathrm{D}_{2}$-dopamine agonist depends upon catecholaminergic neurons. Eur. J. Pharmacol. 113: 109-114.

Breese, G. R., and T. D. Traylor (1970) Effects of 6-hydroxydopamine on norepinephrine and dopamine: Evidence for selective degeneration of catecholamine neurons. J. Pharmacol. Exp. Ther. 174: 413-420.

Breese, G. R., and T. D. Traylor (1972) Developmental characteristics of brain catecholamines and tyrosine hydroxylase in the rat: Effects of 6-hydroxydopamine. Br. J. Pharmacol. 44: 210-222.

Breese, G. R., R. D. Smith, and B. R. Cooper (1975) Effect of various 6-hydroxydopamine treatments during development on growth and ingestive behavior. Pharmacol. Biochem. Behav. 3: 1097-1 106.

Breese, G. R., A. A. Baumeister, T. J. McCown, S. G. Emerick, G. D. Frye, K. Crotty, and R. A. Mueller (1984) Behavioral differences between neonatal and adult-6-hydroxydopamine treated rats to dopamine agonists: Relevance to neurological symptoms in clinical syndromes with reduced brain dopamine. J. Pharmacol. Exp. Ther. 231: 343-354.

Breese, G. R., A. Baumeister, T. C. Napier, G. D. Frye, and R. A. Mueller (1985a) Evidence that D-1 dopamine receptors contribute to the supersensitive behavioral responses induced by L-dihydroxyphenylalanine in rats treated neonatally with 6-hydroxydopamine. J. Pharmacol. Exp. Ther. 235: 287-295.

Breese, G. R., T. C. Napier, and R. A. Mueller (1985b) Dopamine agonist-induced locomotor activity in rats treated with 6-hydroxydopamine at differing ages: Functional supersensitivity of D-1 dopamine receptors in neonatally lesioned rats. J. Pharmacol. Exp. Ther. 234: 447-455.

Breese, G. R., R. A. Mueller, T. C. Napier, and G. E. Duncan (1986) Neurobiology of $D_{1}$-dopamine receptors after neonatal-6-OHDA treatment: Relevance to Lesch-Nyhan disease. Adv. Exp. Med. Biol. 204: 197-215.

Breese, G. R., G. E. Duncan, T. C. Napier, S. C. Bondy, L. C. Iorio, and R. A. Mueller (1987) 6-Hydroxydopamine treatments enhance behavioral responses to intracerebral microinjection of $D_{1}$ and $D_{2-}$ dopamine agonists into nucleus accumbens and striatum without changing dopamine antagonist binding. J. Pharmacol. Exp. Ther. 240: 107-176.

Bruno, J. P., E. M. Striker, and M. J. Zigmond (1985) Rats given dopamine-depleting brain lesions as neonates are subsensitive to dopaminergic antagonists as adults. Behav. Neurosci. 99: 771-775. 
Coward, D. M. (1986) Apomorphine response plasticity in lesioned rats: Supersensitivity dependency and lack of drug- or non-drug-associated environmental cuing. Psychopharmacology 90: 253-258.

Criswell, H. E., R. A. Mueller, and G. R. Breese (1988) Assessment of purine-dopamine interactions in 6-hydroxydopamine lesioned rats: Evidence for pre- and postsynaptic influences by adenosine. J. Pharmacol. Exp. Ther. 244: 493-500.

Duncan, G. E., H. E. Criswell, T. J. McCown, I. A. Paul, R. A. Mueller, and G. R. Breese (1987) Behavioral and neurochemical responses to haloperidol and SCH-23390 in rats treated neonatally or as adults with 6-hydroxydopamine. J. Pharmacol. Exp. Ther. 243: 1027-1038.

Hollister, A. S., G. R. Breese, and B. R. Cooper (1974) Comparison of tyrosine hydroxylase and dopamine-B-hydroxylase inhibition with the effects of various 6-hydroxydopamine treatments on d-amphetamine induced motor activity. Psychopharmacologia 36: 1-16.

Iorio, L. C., A. Barnett, F. H. Leitz, V. P. Houser, and A. Korduba (1983) SCH-23390, a potential benzazepine antipsychotic with unique interactions on dopaminergic systems. J. Pharmacol. Exp. Ther. 226: $462-468$.

Kilts, C. D., G. R. Breese, and R. B. Mailman (1981) Simultaneous quantifaction of dopamine, 5-hydroxytryptamine, and four metabolically related compounds by means of reverse phase HPLC with electrochemical detection. J. Chromatogr. Biol. Med. Appl. 225:347357.

Klawans, H. L., and D. I. Margolins (1975) Amphetamine-induced dopaminergic hypersensitivity in guinea pigs. Arch. Gen. Psychiatry 22: 725-732.

Morelli, M., and G. Di Chiara (1987) Agonist-induced homologous and heterologous sensitization to D-1- and D-2-dependent contraversive turning. Eur. J. Pharmacol. 141: 101-107.

Morelli, M., S. Fenu, and G. Di Chiara (1987) Behavioral expression of $D-1$ receptor supersensitivity depends on previous stimulation of D-2 receptors. Life Sci. 40: 245-251.

Ondrusek, M. G., C. D. Kilts, G. D. Frye, R. B. Mailman, R. A. Mueller, and G. R. Breese (1981) Behavioral and biochemical studies of the scopolamine-induced reversal of neuroleptic activity. Psychopharmacology 73: 17-22.

Paxinos, G., and C. Watson (1982) The Rat Brain in Stereotaxic Coordinates, Academic, New York.

Post, R. M. (1980) Intermittent versus continuous stimulation: Effect of time interval on the development of sensitization or tolerance. Life Sci. 26: 1275-1282.

Robinson, T. E. (1984) Behavioral sensitization: Characterization of enduring changes in rotational behavior produced by intermittent injections of amphetamine in male and female rats. Psychopharmacology 84: 466-475

Robinson, T. E., and J. B. Becker (1986) Enduring changes in brain and behavior produced by chronic amphetamine administration: A review and evaluation of animal models of amphetamine psychosis. Brain Res. Rev. 11: 157-198.

Smith, R. D., B. R. Cooper, and G. R. Breese (1973) Growth and behavioral changes in developing rats treated intracisternally with 6-hydroxydopamine: Evidence for involvement of brain dopamine. J. Pharmacol. Exp. Ther. 185: 609-613.

Stoof, J. C., and J. W. Kebabian (1984) Two dopamine receptors: Biochemistry, physiology and pharmacology. Life Sci. 35: 2281-2286.

Tilson, H. A., and R. H. Rech (1973) Conditioned drug effects and absence of tolerance to d-amphetamine induced motor activity. Pharmacol. Biochem. Behav. I: 149-153.

Tsuruta, K., E. A. Frey, C. W. Grewe, T. E. Cote, R. L. Eskay, and T. W. Kebabian (1981) Evidence that LY-141865 specifically stimulates the D-2 dopamine receptor. Nature 292: 463-465.

Widerlov, E., C. D. Kilts, R. B. Mailman, R. B. Nemeroff, A. J. Prang, and G. R. Breese (1982) Increase in dopamine metabolites in rat brain by neurotensin. J. Pharmacol. Exp. Ther. 221: 1-6.

Winer, R. J. (1971) Statistical Principles in Experimental Design, McGraw-Hill, New York. 